\title{
Lattice gauge theories beyond QCD
}

\author{
Agostino Patella* \\ CERN and Plymouth University \\ E-mail: agostino.patellaecern.ch
}

As the number of flavours in QCD is increased beyond some threshold, chiral symmetry is restored and long-distance physics becomes scale invariant. The region of parameter space in which this happens is called "conformal window". The phenomenology of theories in the conformal window or close to it is very different from real-world QCD, and clearly shows how rich gauge theories can be. Near-conformal gauge theories have been conjectured to be viable models for physics beyond the Standard Model, and as they might naturally incorporate a Higgs-like scalar, and in this sense they are very different from traditional techicolor theories. As these theories are inherently non-perturbative, lattice simulation provide a unique setup to investigate them from first principles. In this talk I will review the main results and open questions in this field, the challenges ahead, and the techniques used.

Frontiers of Fundamental Physics 14 - FFP14,

15-18 July 2014

Aix Marseille University (AMU) Saint-Charles Campus, Marseille

\footnotetext{
*Speaker.
} 